Article

\title{
Surface Modification of Sol-Gel Silica Antireflective Coatings by F-PMHS: A Simple Method for Improvement of Amphiphobicity
}

\author{
Wensheng Lin ${ }^{1,+}{ }^{+}$, Yingying Sun ${ }^{1, \dagger}{ }^{\dagger}$ Jiaxian Zheng ${ }^{1}$, Yanmei Zheng ${ }^{1}$, Lianghong Yan ${ }^{2}$, \\ Bo Jiang ${ }^{3}$, Wenbin Yang ${ }^{1}$, Hanxian Chen ${ }^{1, *}$ and Xinxiang Zhang ${ }^{1, *(D)}$ \\ 1 College of Materials Engineering, Fujian Agriculture and Forestry University, Fuzhou 350108, China; \\ wensheng0817@163.com (W.L.); 18065167449@163.com (Y.S.); zjx15505909116@163.com (J.Z.); \\ SEUElaine@163.com (Y.Z.); fafuywb@163.com (W.Y.) \\ 2 Research Center of Laser Fusion, China Academy of Engineering Physical, Mianyang 621900, China; \\ yanlianghong@126.com \\ 3 Key Laboratory of Green Chemistry \& Technology, College of Chemistry, Sichuan University, \\ Chengdu 610064, China; jiangbo@scu.edu.cn \\ * $\quad$ Correspondence: hanxian1229@163.com (H.C.); xxzhang0106@163.com (X.Z.); Tel.: +86-591-8371-5175 (X.Z.) \\ + These authors contributed to this work equally.
}

Received: 23 December 2017; Accepted: 16 January 2018; Published: 5 February 2018

\begin{abstract}
Sol-gel silica antireflective coatings (ARCs) with improved amphiphobicity were simply fabricated on BK7 glass substrates via fluorinated-poly(methylhydrogen)siloxane (F-PMHS) surface modification by the dip-coating method. The results of Fourier Transform Infrared (FTIR) and X-ray Photoelectron Spectroscopy (XPS) showed that F-PMHS were covalently bonded to the surface of ARCs. F-PMHS modification significantly improved hydrophobicity and oleophobicity of silica ARCs by increasing their water contact angles from $27^{\circ}$ to $105^{\circ}$ and oil contact angles from $17^{\circ}$ to $45^{\circ}$. In addition to the improved amphiphobicity, the modified ARCs also possessed excellent transmittance. Most importantly, it was found that with increasing F-PMHS content the atom amounts and porous property of modified ARCs were almost unchanged. This result had been shown to be associated with the changes of optical property and amphiphobicity for silica ARCs, and the details were discussed.
\end{abstract}

Keywords: amphiphobicity; surface modification; sol-gel method; antireflective coating; fluorinated-poly(methylhydrogen)siloxane

\section{Introduction}

Sol-gel silica antireflective coatings (ARCs) have captured a considerable amount of attention in the past few decades, since they exhibit outstanding properties, such as superior homogeneity, controllable structure, adjustable refractive index, and have a wide variety of potential applications in solar panels, light sensors, and high-powered laser fusion systems [1-6]. However, sol-gel silica ARCs are usually porous and polar and apt to absorb contamination from the working environment [7]. This leads to gradual decrease in transmittance of sol-gel silica ARCs. This phenomenon is generally called poor AR stability. Sol-gel silica ARCs are the most commonly used ARCs in high-powered laser fusion systems, in which there are thousands of transmissive optics. An obvious decrease in transmittance will seriously decrease the final output energy of lasers. Therefore, the AR stability is very important for sol-gel silica ARCs used in high-powered laser fusion systems. It was reported that the hydrophobic modification of sol-gel silica ARCs was an effective method for improving their AR stability [8]. Many scholars have reported different methods to prepare hydrophobic ARCs [9-12]. These hydrophobic sol-gel silica ARCs show good hydrophobicity and AR stability to hydrophilic 
pollutants. However, the hydrophobic modification of sol-gel silica ARCs incorporates a great number of hydrophobic groups into the silica. These hydrophobic groups make the modified ARCs difficult to absorb polar contaminants, but easy to absorb organic pollutants $[13,14]$. There are also some oleophilic pollutants in high-powered laser fusion systems and, therefore, it is desirable to improve the amphiphobicity of sol-gel silica ARCs to afford them good AR stability to hydrophilic and oleophilic pollutants simultaneously.

In our previous work [15], PMHS surface modification was reported as an ultra-fast and effective method for hydrophobic modification of sol-gel silica ARCs. However, the PMHS-modified ARCs also suffer from poor AR stability to the oleophilic pollutants. In this work, surface modification of sol-gel silica ARCs by F-PMHS was reported to improve the hydrophobicity and oleophobicity simultaneously. The resultant F-PMHS modified ARCs exhibited not only improved amphiphobicity, but also good transmittance.

\section{Materials and Methods}

\subsection{Mateirals}

High purity tetraethyl orthosilicate (TEOS) was purchased from Kermel (Tianjin, China). Analytical grade ammonia-water with an $\mathrm{NH}_{3}$ content of $25-28 \%$ was purchased from Sinopharm Chemical Reagent Co., Ltd., Shanghai, China. Analytical-grade ethanol and hexane were purchased from Tianjin Zhiyuan Chemical Reagent Co., Ltd., Tianjin, China. F-PMHS and Kastredt catalyst (platinum-1,3-divinyl-1,1,3,3-tetramethyldisiloxane) were purchased from Chengguang Research Institute of Chemical Industry (Chengdu, China).

\subsection{Fabrication of Silica Sols}

TEOS, ethanol and ammonia-water were added in a sealed glass container and then magnetically agitated for $2 \mathrm{~h}$ at $30{ }^{\circ} \mathrm{C}$. Then, the sol was aged at $25{ }^{\circ} \mathrm{C}$ for 7 days. The molar ratio of TEOS: $\mathrm{NH}_{3}: \mathrm{EtOH}: \mathrm{H}_{2} \mathrm{O}$ was 1:0.73:37.74:1.98.

\subsection{Preparation of Sol-Gel Silica ARCS}

BK7 glass substrates with a diameter of $30 \mathrm{~mm}$ and thickness of $3 \mathrm{~mm}$ were dipped into $0.5 \% \mathrm{HF}$ solution for several seconds and then immediately washed with deionized water. Finally, the substrates were washed with a hydrous ethanol and wiped carefully with a cleanroom wiper. Silica ARCs were obtained by dipping BK7 glass substrates in sols and subsequently withdrawing at a constant speed of $100 \mathrm{~mm} / \mathrm{min}$. The obtained ARCs were heated at $120{ }^{\circ} \mathrm{C}$ for $1 \mathrm{~h}$. In this work, the dip-coater (SYDC-100N) was purchased from SANYAN Instrument Co., Ltd. (Shanghai, China). The humidity was well controlled to $40 \%$ with a nitrogen atmosphere.

\subsection{Surface Modification of Silica ARCs}

The modifier solutions with weight ratio of F-PMHS of $0.125 \%, 0.25 \%, 0.5 \%, 0.75 \%, 1.0 \%$, and $1.25 \%$ were obtained by mixing hexane, F-PMHS and Kastredt catalyst together. The F-PMHS surface-modified silica ARCs were fabricated by immersing the sol-gel silica ARCs into the modifier solution and immediately removing at a withdrawal rate of $100 \mathrm{~mm} / \mathrm{min}$. The modified ARCs were flushed with hexane to remove unreacted F-PMHS and then heated at $120^{\circ} \mathrm{C}$ for $1 \mathrm{~h}$.

\subsection{Characterization}

The xerogel for FTIR characterization was obtained by rotary evaporation with a further heat-treatment at $120{ }^{\circ} \mathrm{C}$ for $1 \mathrm{~h}$. The F-PMHS-modified xerogel was realized by immersing xerogel into the modifier solution with a weight ratio of F-PMHS of $0.5 \%$ for $1 \mathrm{~min}$. The F-PMHS-modified xerogel was heat-treated at $120{ }^{\circ} \mathrm{C}$ for $1 \mathrm{~h}$ and then extracted with hexane for $48 \mathrm{~h}$ to remove F-PMHS which does not covalently bond to silica particles. FTIR spectra of xerogels before and after F-PMHS 
modification were analyzed by a Bruker Tensor 27 (Bruker Optik, Ettlingen, Germany) using the KBr pellet method with transmission mode in the mid-IR range from 500 to $4000 \mathrm{~cm}^{-1}$.XPS spectra of AR coatings were obtained by using a Thermo SCIENTIFIC ESCALAB 250Xi (Thermo Fisher Scientific, Pittsburgh, PA, USA) with AlX-radiation $(\mathrm{K} \alpha, \mathrm{h} v=1486.8 \mathrm{eV})$. The water and oil contact angles (WCA and OCA) of ARCs were obtained on a Krüss DSA100 (Hamburg, Germany). The surface morphology of the coating was analyzed by scanning electron microscope (SEM, ZEISS Z500, Jena, Germany). The refractive index and film thickness of the ARCs were determined using an ellipsometer at $633 \mathrm{~nm}$ (Horiba UVISEL, Longjumeau, France). The transmittance spectra were measured with a UV-VIS spectrophotometer (Mapada, 3200PC, Shanghai, China).

\section{Results and Discussion}

\subsection{FTIR Characterization}

FTIR spectra of silica xerogels before and after F-PMHS modification were recorded and represented in Figure 1. Before FTIR characterization, the modified silica xerogel has been extracted with hexane for $48 \mathrm{~h}$ to remove F-PMHS which did not covalently bind to the silica particles. For pure silica xerogel, there are absorption bands observed at around $3450 \mathrm{~cm}^{-1}, 2964 \mathrm{~cm}^{-1}, 1630 \mathrm{~cm}^{-1}$, $1090 \mathrm{~cm}^{-1}, 954 \mathrm{~cm}^{-1}$, and $800 \mathrm{~cm}^{-1}$. The broad absorption band at around $3450 \mathrm{~cm}^{-1}$ is attributed to $\mathrm{Si}-\mathrm{OH}$ bonds or absorbed water $[16,17]$. The three peaks around $2964 \mathrm{~cm}^{-1}$ corresponds to the stretching vibration of $\mathrm{C}-\mathrm{H}$ bonds from $-\mathrm{CH}_{3}$ [18]. The water deformation band appear at about $1630 \mathrm{~cm}^{-1}$ [19]. The absorption bands at around $1090 \mathrm{~cm}^{-1}$ and $800 \mathrm{~cm}^{-1}$ are the characteristic absorption band of $\mathrm{Si}-\mathrm{O}-\mathrm{Si}$ bond due to the asymmetric and symmetric vibration, respectively $[20,21]$. The absorption band at $961 \mathrm{~cm}^{-1}$ is attributed to -Si-OH [22]. After F-PMHS surface modification, there are two new absorption bands at $900 \mathrm{~cm}^{-1}$ and $2167 \mathrm{~cm}^{-1}$ corresponding to $-\mathrm{Si}-\mathrm{C}$ and $-\mathrm{Si}-\mathrm{H}$ of F-PMHS, respectively [23]. In addition, the intensity of absorption band at $2964 \mathrm{~cm}^{-1}$ is strengthen while that of absorption band at $961 \mathrm{~cm}^{-1}$ is weakened gradually with the increasing F-PMHS concentration. The latter demonstrates convincingly that $-\mathrm{Si}-\mathrm{H}$ groups of F-PMHS react with the hydroxyl groups of silica particles by dehydrogenation and, hence, F-PMHS chains are covalently grafted onto the surface of silica particles.

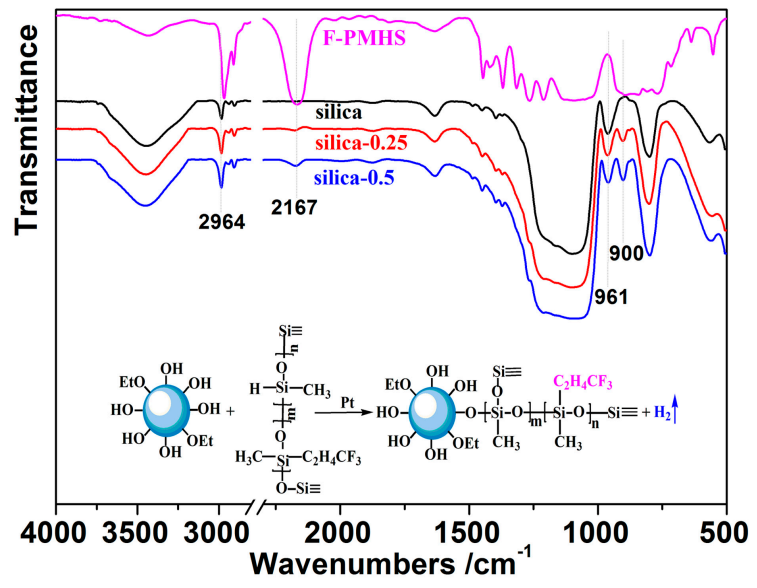

Figure 1. FTIR spectra of PMHS, pure silica xerogel, and xerogel modified with F-PMHS content of $0.25 \%$ and $0.5 \%$.

\subsection{XPS Analysis}

It is difficult to demonstrate the existence of amphiphobic- $\mathrm{CF}_{3}$ groups by FTIR because its absorption bands are similar to those of $-\mathrm{CH}_{3}$ [23]. So the characterization of XPS is carried out further. And before XPS characterization, the modified $\mathrm{SiO}_{2} \mathrm{ARC}$ s were washed with hexane three times to 
remove unreacted F-PMHS. XPS spectra of ARCs and corresponding atomic amounts of $\mathrm{O}, \mathrm{Si}, \mathrm{C}$, and $\mathrm{F}$ as a function of F-PMHS content are represented in Figure 2a,b. As shown in Figure 2a, the ARCs consists of $\mathrm{O}, \mathrm{Si}$, and $\mathrm{C}$ elements before F-PMHS modification [24,25], while there is an additional F element after F-PMHS modification [26]. As shown in Figure 2b, the atomic amounts of $C$ and $F$ for silica ARCs are $13.35 \%$ and 0 , respectively, which are increased to $21.53 \%$ and $10.71 \%$ after being modified by $0.125 \%$ F-PMHS. At the same time, the atomic amounts of $\mathrm{O}$ and Si decrease from $63.43 \%$ and $23.22 \%$ to $47.4 \%$ and $20.07 \%$, respectively. This is in good agreement with the results from FTIR. It is the F-PMHS grafted onto the ARCs that results in this change in atomic amounts.
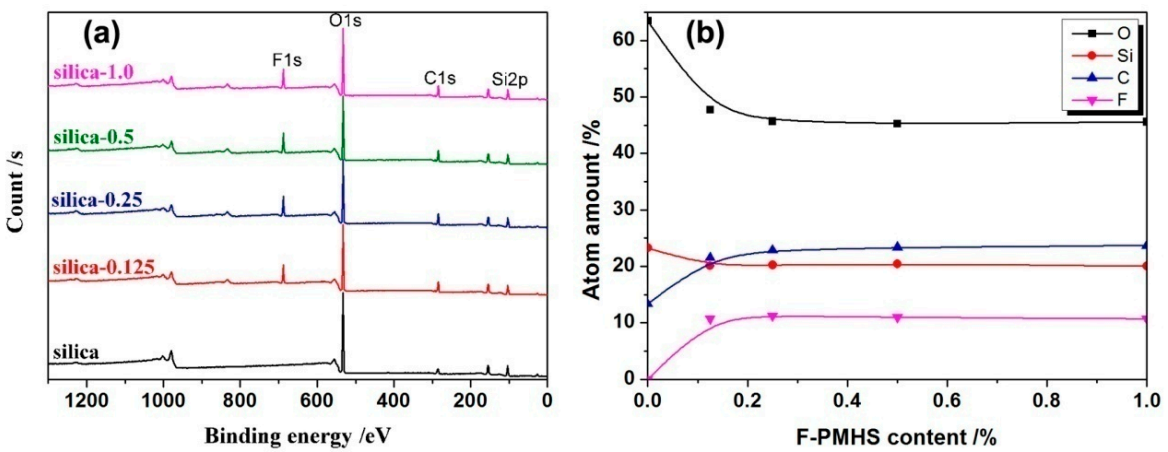

Figure 2. XPS spectra of silica ARC before and after F-PMHS modification (a), and the effect of F-PMHS content on the atom amounts of ARCs (b).

Most importantly, a very interesting phenomenon can be found in Figure $2 \mathrm{~b}$. The atomic amounts are very different before and after F-PMHS modification; however, they are almost unchanged for modified ARCs, no matter what the F-PMHS content in modifier solution is. This can be explained from the ultra-fast reaction between F-PMHS and silica particles. As shown in the schematic representation in Figure 3, sol-gel silica ARC consists of silica particles which randomly stack on the substrate [27]. Silica AR coating is polar and abundant in hydroxyl groups. As long as ARCs being immerged into the modifier solution which contains F-PMHS and Karstedt catalyst, the dehydrogenation happens immediately. Thus, even if at very low F-PMHS content, the surface of porous silica ARC would be easily modified by F-PMHS. With increasing F-PMHS content, the penetration deep of modification also increases. However, the deep modification has no contribution to the XPS characterization because the testing deep of XPS is only $2-10 \mathrm{~nm}$ [28]. This is the reason that the atom amounts for modified ARC is unchanged.

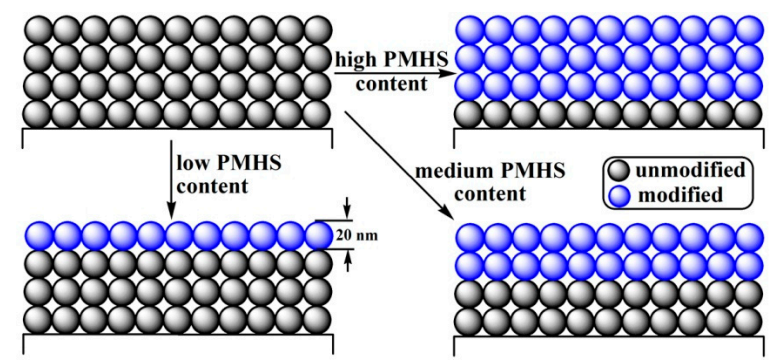

Figure 3. Schematic representation of F-PMHS modification at different F-PMHS content.

\subsection{Morphology of ARCs}

It is very important to investigate the morphology of ARCs before and after F-PMHS surface modification. Figure 4 shows the top-view and cross-sectional SEM images of ARCs modified by F-PMHS with increasing content. It can be found from the top view that the unmodified sol-gel silica ARC is porous. This is benefit for sol-gel silica ARCs because the nano-pores lower the refractive 
index of ARCs to the square root of that of substrate and, therefore, nearly $100 \%$ transmittance can be realized [29,30]. Suppose that, during F-PMHS modification of ARCs, most of the nano-pores in ARCs are replaced by F-PMHS chains, the porosity of sol-gel silica ARCs would be decreased obviously, which would result in a remarkable reduction of transmittance [31]. However, fortunately, the top view shows that the modified ARCs are also porous, indicating that the F-PMHS surface modification does not significantly affect the pore structure of ARCs. It can be also revealed from the cross-sectional SEM images that the thickness of ARCs does not increase significantly after F-PMHS modification.

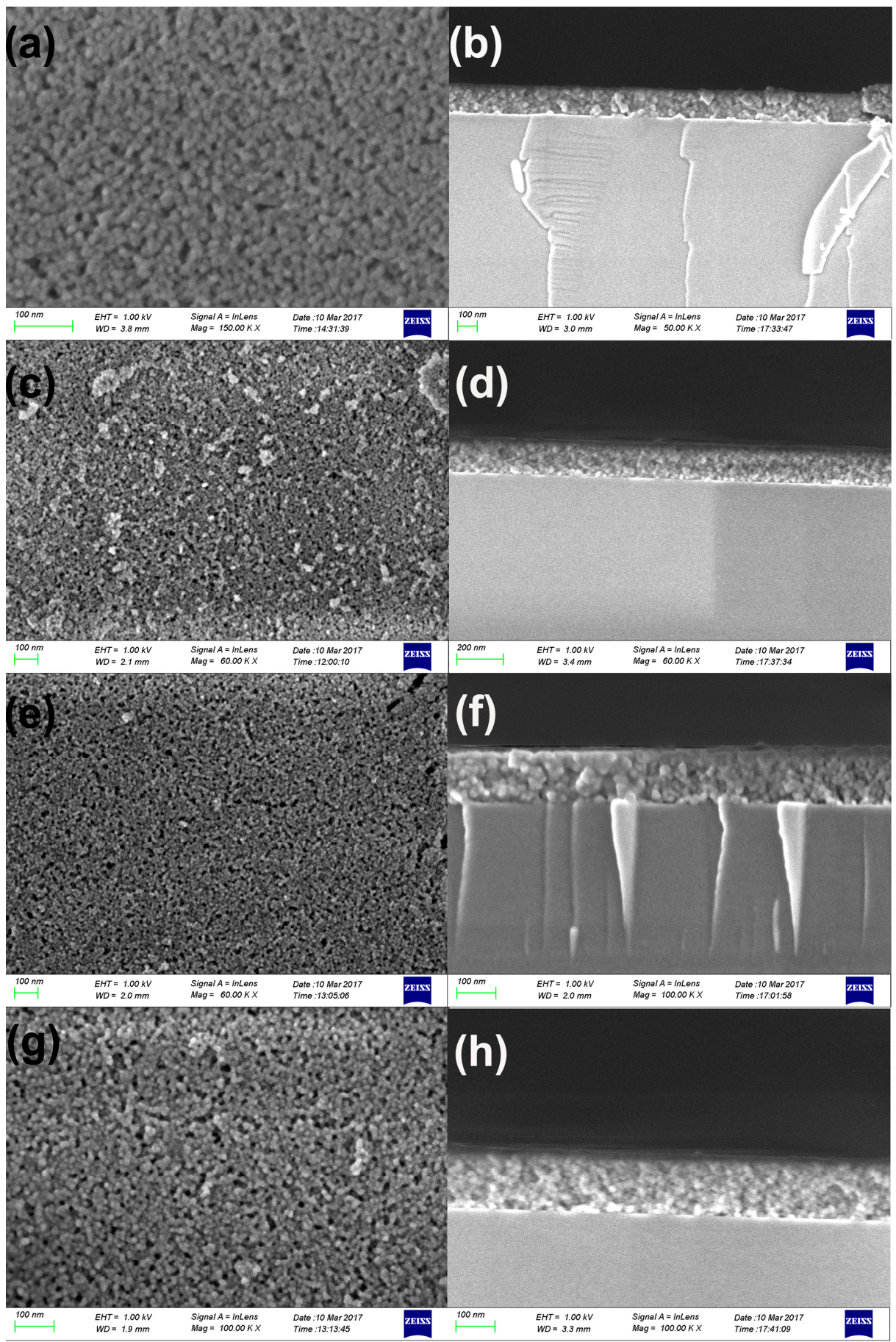

Figure 4. Top-view and cross-sectional SEM images of ARC modified F-PMHS at content of $0.0 \%(\mathbf{a}, \mathbf{b})$, $0.25 \%(\mathbf{c}, \mathbf{d}), 0.5 \%(\mathbf{e}, \mathbf{f})$, and $1.0 \%(\mathbf{g}, \mathbf{h})$. 


\subsection{Hydrophobicity and Oleophobicity}

According to the FTIR and XPS analysis, the chemical composition is quite different for ARCs before and after F-PMHS modification. There are amounts of hydrophobic $-\mathrm{CH}_{3}$ and many amphiphobic- $\mathrm{CF}_{3}$ groups incorporated onto the surface of ARCs. Therefore, the wettability of sol-gel silica ARCs can be adjusted by F-PMHS modification. It is well-known that WCA and OCA measurements can be applied to assess the wettability properties of silica ARCs [32]. Figure 5 shows the WCA and OCA of silica ARCs with and without F-PMHS modification. The WCA and OCA of pure silica ARC is $27^{\circ}$ and $17^{\circ}$, respectively, indicating that it is very hydrophilic and oleophilic. After modification, the WCA and OCA increase significantly to about $105^{\circ}$ and $45^{\circ}$, respectively. In agreement with the XPS analysis, the WCA and OCA does not change with increasing F-PMHS content because the surface chemical property is unchanged.

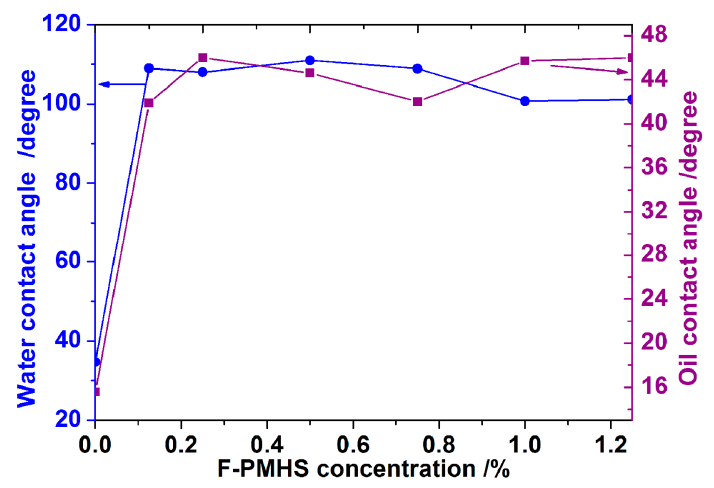

Figure 5. Effect of F-PMHS content on the water and oil contact angles of ARCs.

\subsection{Optical Property of ARCs}

The transmittance spectra of sol-gel silica ARCs before and after modification with different F-PMHS content were recorded and shown in Figure 6. As shown in Figure 6, the central wavelength shifts to the longer wavelength, while the maximum transmittance at central wavelength decrease slightly after F-PMHS modification. The change in maximum transmittance and central wavelength is named as $\Delta T_{\max }$ and $\Delta \lambda$. The effect of F-PMHS content on $\Delta T_{\max }$ and $\Delta \lambda$ can be also read from Figure 6. The $\Delta T_{\max }$ and $\Delta \lambda$ increases gradually with increasing F-PMHS content. The relationship between reflection and refractive index of ARCs is as follows Equation (1) [33]:

$$
R=\left(n_{\mathrm{c}}^{2}-n_{\mathrm{s}}\right)^{2} /\left(n_{\mathrm{c}}{ }^{2}+n_{\mathrm{s}}\right)^{2}=(1-T) / 2
$$

where $n_{\mathrm{C}}$ and $n_{\mathrm{S}}$ are the refractive indices of ARC and substrate, respectively; and $R$ and $T$ are the reflection and transmittance of ARC.

The change in refractive index, thickness and porosity as a function of F-PMHS was showed in Table 1. The refractive index of sol-gel silica ARC is 1.25 to give nearly $100 \%$ transmittance. After being immerging in modifier solutions with increasing F-PMHS concentration, the refractive index of modified ARCs increases gradually from 1.25 to 1.32. This leads to the decrease in transmittance and, hence, an increase in $\Delta T_{\max }$. After being immersed into the modifier solutions with higher F-PMHS content, more F-PMHS chains penetrate into the pores of ARC. The F-PMHS chains partially replace the air in the pores, resulting in a decrease in porosity and a corresponding increase in refractive index. However, the $\Delta T_{\max }$ is small and, therefore, the transmittance of modified ARC is still very high. For example, for ARC modified by 0.5\% F-PMHS, the ARC coated glass exhibits much better readability for the characters below it because of its antireflective property at visible wavelengths. This is in agreement with the discussion in morphology characterization. 
The relationship between central wavelength, refractive index and physical thickness of ARC can refer to Equation (2) [34]:

$$
\lambda=4 n_{\mathrm{c}} d
$$

where $n_{\mathrm{c}}$ and $d$ are the refractive and physical thickness of ARC, respectively.

The increase in refractive index is one of the reasons for the increase of central wavelength. On the other hand, during modification, the surface of silica AR coating was grafted with F-PMHS chains. This will probably increase the physical thickness of ARC to a certain extent. Both of these factors lead to an increase in $\Delta \lambda$ as a function of F-PMHS concentration.

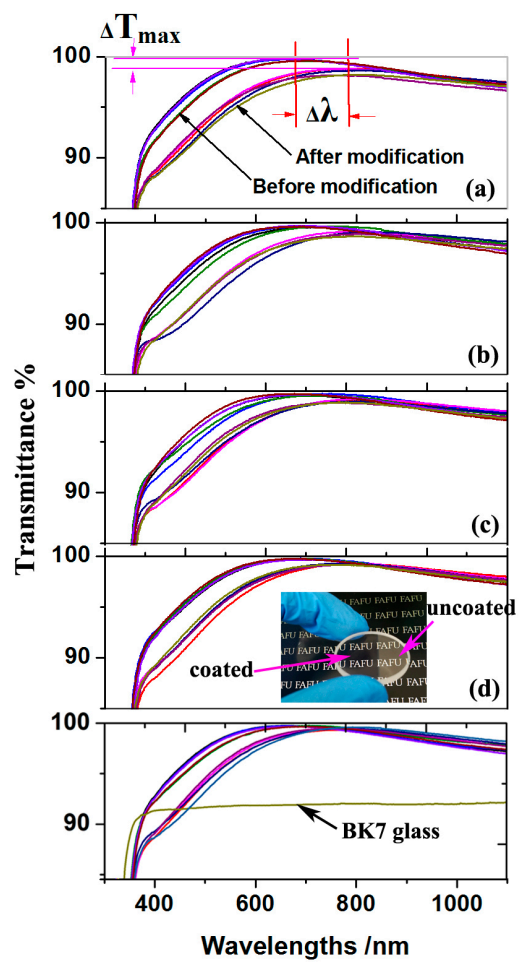

Figure 6. Transmittance spectra of ARCs before and after being modified by F-PMHS in different content of $1.25 \%$ (a), $1.00 \%$ (b), $0.75 \%$ (c), $0.50 \%$ (d), and $0.25 \%(\mathbf{e})$.

Table 1. Change in refractive index, thickness and porosity as a function of F-PMHS concentration.

\begin{tabular}{cccc}
\hline F-PMHS Concentration/\% & Refractive Index & Thickness/nm & Porosity/\% \\
\hline 0 & 1.25 & 136 & 50.3 \\
0.25 & 1.28 & 140 & 43.6 \\
0.5 & 1.29 & 142 & 41.3 \\
0.75 & 1.30 & 141 & 39.0 \\
$1.0 \%$ & 1.31 & 140 & 36.7 \\
1.25 & 1.32 & 141 & 34.4 \\
\hline
\end{tabular}

\section{Conclusions}

In summary, we present a simple route to improve the amphiphobicity of sol-gel silica ARCs. The surface modification process can be achieved simply by immersing silica ARCs into the modifier solution and then immediately withdrawing. The hydrophilic ARCs can be easily switched to hydrophobic after F-PMHS modification and its oleophobicity was improved simultaneously. In addition, modified ARCs also possess good transmittance. The resultant F-PMHS modified sol-gel silica ARCs may find important application in high-powered laser fusion system due to its amphiphobic property and high transmittance. 
Acknowledgments: The authors gratefully acknowledge the support from the National Natural Science Foundation of China (61505029), Natural Science Foundation of Fujian Province of China (2015J05029), and the Outstanding Youth Fund of Fujian Agriculture and Forestry University (XJQ201602).

Author Contributions: Xinxiang Zhang and Hanxian Chen conceived and designed the experiments; Jiaxian Zheng and Yanmei Zheng performed the experiments; Lianghong Yan, Wensheng Lin, and Yingying Sun analyzed the data; Bo Jiang and Wenbin Yang contributed analysis tools; and Wensheng Lin and Yingying Sun wrote the paper. All authors critically revised the article.

Conflicts of Interest: The authors declare no conflict of interest.

\section{References}

1. Hsu, C.C.; Lan, W.L.; Chen, N.P.; Wu, C.C. The hydrophobic and omnidirectional antireflection coating of $\mathrm{SiO}_{2}$ nanospheres with $\mathrm{C}_{18}$-TEOS. Opt. Laser Technol. 2014, 58, 202-206. [CrossRef]

2. Sim, D.M.; Choi, M.J.; Hur, Y.H.; Nam, B.; Chae, G.; Park, J.H.; Jung, Y.S. Ultra-high optical transparency of robust, graded-index, and anti-fogging silica coating derived from Si-containing block copolymers. Adv. Opt. Mater. 2013, 1, 428-433. [CrossRef]

3. Guo, Z.L.; Zhao, H.X.; Zhao, W.; Wang, T.; Kong, D.P.; Chen, T.J.; Zhang, X.Y. High-quality hollow closed-pore silica antireflection coatings based on styrene-acrylate emulsion@ organic-inorganic silica precursor. ACS Appl. Mater. Interfaces 2016, 8, 11796-11805. [CrossRef] [PubMed]

4. Moghal, J.; Kobler, J.; Sauer, J.; Best, J.; Gardener, M.; Watt, A.A.; Wakefield, G. High-performance, single-layer antireflective optical coatings comprising mesoporous silica nanoparticles. ACS Appl. Mater. Interfaces 2012, 4, 854-859. [CrossRef] [PubMed]

5. Wang, W.T.; Lu, N.; Hao, J.Y.; Xu, H.B.; Qi, D.P.; Chi, L.F. Self-assembled monolayer islands masked chemical etching for broad-band antireflective silicon surfaces. J. Phys. Chem. C 2010, 114, 1989-1995. [CrossRef]

6. Poirié, T.; Schmitt, T.; Bousser, E.; Vernhes, R.; Martinu, L.; Klemberg-Sapieha, J.E. Hybrid organic/inorganic nanolaminate structures with enhanced tribo-mechanical properties for optical applications. Surf. Coat. Technol. 2017, 315, 399-407. [CrossRef]

7. Thomas, I.M.; Burnham, A.K.; Ertel, J.R. Method for reducing the effect of environmental contamination of sol-gel optical coatings. SPIE 1999, 3492, 220-229. [CrossRef]

8. Cai, S.; Xue, Q.L.; Xia, B.B.; Yang, J.; Lv, H.B.; Yan, H.W.; Jiang, B. Hydrophobic-oleophobic antireflective film with excellent optical property prepared by a simple sol-gel route. Mater. Lett. 2015, 156, 14-16. [CrossRef]

9. Manca, M.; Cannavale, A.; De Marco, L.; Arico, A.S.; Cingolani, R.; Gigli, G. Durable superhydrophobic and antireflective surfaces by trimethylsilanized silica nanoparticles-based sol-gel processing. Langmuir 2009, 25, 6357-6362. [CrossRef] [PubMed]

10. Tao, C.; Yan, H.; Yuan, X.; Yin, Q.; Zhu, J.; Ni, W.; Yan, L.; Zhang, L. Hydrophobic antireflective coatings with ultralow refractive index synthesized by deposition of methylated hollow silica nanoparticles. Mater. Lett. 2016, 183, 374-377. [CrossRef]

11. Xia, B.B.; Luo, J.H.; Li, Y.Y.; Yang, B.W.; Zhang, S.M.; Jiang, B. Preparation of sponge-like porous $\mathrm{SiO}_{2}$ antireflective coatings with excellent environment-resistance by an acid-catalysed sol-gel method. RSC Adv. 2017, 7, 26834-26838. [CrossRef]

12. Li, T.; He, J. A facile hybrid approach to high-performance broadband antireflective thin films with humidity resistance as well as mechanical robustness. J. Mater. Chem. C 2016, 4, 5342-5348. [CrossRef]

13. Arturi, K.R.; Jepsen, H.; Callsen, J.N.; Søgaard, E.G.; Simonsen, M.E. Superhydrophilicity and durability of fluoropolymer- $\mathrm{TiO}_{2}$ coatings. Prog. Organ. Coat. 2016, 90, 132-138. [CrossRef]

14. Shabnam, R.; Ahmad, H. Hydrophobic poly(lauryl methacrylate)-coated magnetic nano-composite particles for removal of organic pollutants. Polym. Adv. Technol. 2015, 26, 408-413. [CrossRef]

15. Zhang, X.X.; Xia, B.B.; Ding, B.; Zhang, Y.L.; Luo, J.H.; Jiang, B. Ultra-fast surface hydrophobic modification of sol-gel silica antireflective coating with enhanced abrasion-resistance. Mater. Lett. 2013, 104, 31-33. [CrossRef]

16. Ganbavle, V.V.; Bangi, U.K.H.; Latthe, S.S.; Mahadik, S.A.; Rao, A.V. Self-cleaning silica coatings on glass by single step sol-gel route. Surf. Coat. Technol. 2011, 205, 5338-5344. [CrossRef]

17. Mosquera, M.J.; de los Santos, D.M.; Rivas, T. Surfactant-synthesized ormosils with application to stone restoration. Langmuir 2010, 26, 6737-6745. [CrossRef] [PubMed] 
18. Zhang, G.W.; Lin, S.D.; Wyman, I.; Zou, H.L.; Hu, J.W.; Liu, G.J.; Wang, J.D.; Li, F.; Liu, F.; Hu, M.L. Robust superamphiphobic coatings based on silica particles bearing bifunctional random copolymers. ACS Appl. Mater. Interfaces 2013, 5, 13466-13477. [CrossRef] [PubMed]

19. Vincent, A.; Babu, S.; Brinley, E.; Karakoti, A.; Deshpande, S. Role of catalyst on refractive index tunability of porous silica antireflective coatings by sol-gel technique. J. Phys. Chem. C 2007, 111, 8291-8298. [CrossRef]

20. Sun, J.; Cui, X.; Zhang, C.; Zhang, C.; Ding, R.; Xu, Y. A broadband antireflective coating based on a double-layer system containing mesoporous silica and nanoporous silica. J. Mater. Chem. C 2015, 3, 7187-7194. [CrossRef]

21. Latthe, S.; Liu, S.; Terashima, C.; Nakata, K.; Fujishima, A. Transparent, adherent, and photocatalytic $\mathrm{SiO}_{2}-\mathrm{TiO}_{2}$ coatings on polycarbonate for self-cleaning applications. Coatings 2014, 4, 497-507. [CrossRef]

22. Kuo, T.W.; Wang, N.F.; Tsai, Y.Z.; Hung, P.K.; Houng, M.P. Broadband triple-layer $\mathrm{SiO}_{x} / \mathrm{SiO}_{x} \mathrm{~N}_{y} / \mathrm{SiN}_{x}$ antireflective coatings in textured crystalline silicon solar cells. Mater. Sci. Semicond. Process. 2014, 25, 211-218. [CrossRef]

23. Brassard, J.D.; Sarkar, D.K.; Perron, J. Synthesis of monodisperse fluorinated silica nanoparticles and their superhydrophobic thin films. ACS Appl. Mater. Interfaces 2011, 3, 3583-3588. [CrossRef] [PubMed]

24. Wang, Q.J.; Quan, Y.W.; Zhang, J.S.; Chen, Q.M. Preparation of super water-repellent membrane by radiation-induced copolymerization. Surf. Coat. Technol. 2006, 200, 5493-5497. [CrossRef]

25. Yang, F.C.; Guo, Z.G. Bio-inspired design of a transparent $\mathrm{TiO}_{2} / \mathrm{SiO}_{2}$ composite gel coating with adjustable wettability. J. Mater. Sci. 2016, 51, 7545-7553. [CrossRef]

26. Ren, X.H.; Fan, H.Q.; Ma, J.W.; Wang, C.; Zhao, Y.W.; Lei, S.H. Triboelectric nanogenerators based on fluorinated wasted rubber powder for self-powering application. ACS Sustain. Chem. Eng. 2017, 5, 1957-1964. [CrossRef]

27. Cui, X.M.; Ding, R.M.; Wang, M.C.; Zhang, C.; Zhang, C.; Zhang, J.; Xu, Y. In situ surface assembly derived ultralow refractive index $\mathrm{MgF}_{2}-\mathrm{SiO}_{2}$ hybrid film for tri-layer broadband antireflective coating. Adv. Opt. Mater. 2016, 4, 722-730. [CrossRef]

28. Goussé, C.; Chanzy, H.; Excoffier, G. Stable suspensions of partially silylated cellulose whiskers dispersed in organic solvents. Polymer 2002, 43, 2645-2651. [CrossRef]

29. Kim, S.; Cho, A.; Kim, S.; Cho, W.; Chung, M.H.; Kim, F.S.; Kim, J.H. Multi-purpose overcoating layers based on PVA/silane hybrid composites for highly transparent, flexible, and durable AgNW/PEDOT:PSS films. RSC Adv. 2016, 6, 19280-19287. [CrossRef]

30. Siddique, R.H.; Gomard, G.; Holscher, H. The role of random nanostructures for the omnidirectional anti-reflection properties of the glasswing butterfly. Nat. Commun. 2015, 6, 6909. [CrossRef] [PubMed]

31. Xia, B.B.; Zhang, Q.H.; Yao, S.Y.; Zhang, Y.L.; Xiao, B.; Jiang, B. Sol-gel silica antireflective coating with enhanced abrasion-resistance using polypropylene glycol as porogen. J. Sol-Gel Sci. Technol. 2014, 71, 291-296. [CrossRef]

32. Yao, L.; He, J.H. Broadband antireflective superhydrophilic thin films with outstanding mechanical stability on glass substrates. Chin. J. Chem. 2014, 32, 507-512. [CrossRef]

33. Floch, H.G.; Belleville, P.F. A scratch-resistant single-layer antireflective coating by a low temperature sol-gel route. J. Sol-Gel Sci. Technol. 1994, 13, 293-304. [CrossRef]

34. Sun, Y.Y.; Zheng, J.X.; Huang, R.C.; Zhang, X.X.; Chen, C.X.; Jiang, B.; Chen, H.X.; Yan, L.H.; Yang, W.B. A simple method to control the microstructure and properties of sol-gel silica antireflective coatings. RSC Adv. 2017, 7, 31950-31959. [CrossRef]

(C) 2018 by the authors. Licensee MDPI, Basel, Switzerland. This article is an open access article distributed under the terms and conditions of the Creative Commons Attribution (CC BY) license (http:/ / creativecommons.org/licenses/by/4.0/). 